\title{
Accessing the subterranean ant fauna (Hymenoptera: Formicidae) in native and modified subtropical landscapes in the Neotropics
}

\author{
Mila Ferraz de Oliveira Martins ${ }^{* 1} @$, Marcilio José Thomazini², Dilmar Baretta ${ }^{3}$, George Gardner Brown ${ }^{2}$,
}

Marcio Gonçalves da Rosa ${ }^{3} \mathbb{D}^{\text {, Mauricio Rumenos Guidetti Zagatto }}{ }^{5}$, Alessandra Santos ${ }^{2}$, Herlon Sérgio Nadolny'2, Guilherme Borges Xarão Cardoso ${ }^{2}$, Cintia Carla Niva ${ }^{2,6}$, Marie Luise Carolina Bartz ${ }^{4}$ \& Rodrigo Machado Feitosa ${ }^{I}$

${ }^{1}$ Universidade Federal do Paraná, Avenida Francisco Heráclito dos Santos, s/n, Centro Politécnico, CEP: 81531980, Curitiba, PR, Brasil

${ }^{2}$ Empresa Brasileira de Pesquisa Agropecuária, Estrada da Ribeira, km. 111, Guaraituba, CEP: 83411000, Curitiba, PR, Brasil

${ }^{3}$ Universidade do Estado de Santa Catarina, Centro de Educação Superior do Oeste, Rua Beloni Trombeta Zanin, 680, Santo Antônio, CEP: 89815630, Chapecó, SC, Brasil

${ }^{4}$ Universidade Positivo, Rua Professor Pedro Viriato Parigot de Souza 3841/3842, Cidade Industrial, CEP: 81280330, Curitiba, PR, Brasil

${ }^{5}$ Escola Superior de Agricultura Luiz de Queiroz, Piracicaba, SP, Brasil

${ }^{6}$ Empresa Brasileira de Pesquisa Agropecuária - Embrapa Cerrados Rodovia BR 020

Km18, Brasília, DF, Brasil

*Corresponding author: Mila Ferrazde Oliveira Martins, e-mail: milafomartins@gmail.com

MARTINS, M.F.O., THOMAZINI, M.J., BARETTA, D., BROWN, G.G., ROSA, M.G., ZAGATTO, M.R.G., SANTOS, A., NALDONY, H.S., CARDOSO, G. B.X., NIVA, C.C., BARTZ, M.L.C., FEITOSA, R.M. Accessing the subterranean ant fauna (Hymenoptera: Formicidae) in native and modified subtropical landscapes in the Neotropics. Biota Neotropica. 20(1): e20190782. http://dx.doi.org/10.1590/1676-0611-BN-2019-0782

\begin{abstract}
Several studies have characterized and delimited subterranean ant assemblages. Soil extraction, one of the methods employed to access this fauna, employs the removal of monoliths. One of the most widely used methods for the extraction of soil sampling is called TSBF (developed by the Tropical Soil Biology and Fertility Programme). This method provides relevant data about the species associated with the soil. In the present study we characterized assemblages of subterranean ants using the TSBF method in different subtropical areas of the Neotropics. We considered two sampling designs in different localities. The first design resulted in $315 \mathrm{TSBF}$ samples obtained from layers at distinct depths. The second design resulted in 270 TSBF samples and 270 epigaeic pitfall trap samples. This material was used to delimit the species that occur exclusively in the subterranean stratum (TSBF) and that are not found on the soil surface. A total of 281 species were recorded. Of these, 57 can be considered subterranean, based on their occurrence in subterranean strata. We also verified that the highest occurrence of ants was in the first $10 \mathrm{~cm}$ of soil depth. Due to the importance of using methods that efficiently extract the subterranean ant fauna in studies, we suggest the TSBF method should be used to sample ants or to associate this method with epigaeic pitfall traps to delimit strictly subterranean assemblages in specific community stratification studies.
\end{abstract} Keywords: Soil, hypogaeic, Brazil, monoliths, pitfall.

\section{Avaliação da mirmecofauna subterrânea (Hymenoptera: Formicidae) em paisagens nativas e modificadas na região subtropical do Neotrópico}

Resumo: Vários estudos têm caracterizado e delimitado assembleias de formigas subterrâneas. A extração de solo, um dos métodos utilizados para amostragem dessa fala, implica na remoção de monólitos. Um dos métodos mais utilizados na extração de monólitos é chamado TSBF (desenvolvido pelo Programa Tropical Soil Biology and Fertility). Esse método fornece dados extremamente relevantes sobre as espécies associadas ao solo. No presente estudo, nós caracterizamos as assembleias de formigas subterrâneas usando o método TSBF em diferentes áreas subtropicais do Neotrópico. Utilizamos dois delineamentos amostrais distintos. O primeiro delineamento resultou em 315 amostras de TSBF obtidas a partir da estratificação do solo. O segundo resultou em 270 amostras de TSBF mais 270 provenientes de armadilhas pitfall epigeicas. Esse material foi utilizado para delimitar espécies que ocorrem exclusivamente no estrato subterrâneo (TSBF) e não são encontradas na superfície do solo. Registramos um total de 281 espécies. Destas, 57 podem ser consideradas subterrâneas (baseado na ocorrência no respectivo estrato). Também verificamos que a maior ocorrência de formigas se deu nos primeiros $10 \mathrm{~cm}$ de profundidade. Devido à importância do uso de métodos que coletem eficientemente formigas subterrâneas, sugerimos o uso do método TSBF em estudos de estratificação de comunidades ou sua associação a armadilhas do tipo pitfall epigeica para delimitar assembleias estritamente subterrâneas.

Palavras-chave: Solo, hipogeicas, Brasil, monólitos, pitfall. 


\section{Introduction}

Ants are conspicuous organisms present in most terrestrial environments (Folgarait 1998), from the arboreal to the subterranean strata (Lucky et al. 2013, Jacquemin et al. 2016). However, little is known about underground ant assemblages (Ryder Wilkie et al. 2007, Andersen \& Brault 2010, Jacquemin et al. 2012), mainly because there are few efficient and specific methods for collecting this important fauna (Wong \& Guénard 2017). Underground ants are the last biodiversity frontier to be explored in Formicidae (Rider Wilkie et al. 2007, Andersen \& Brault 2010), and are also important for the understanding of the origin and evolution of Formicidae (Lucky et al. 2013). In addition, subterranean ants are considered bioturbators sensu strictu (Bottinelli et al. 2015), and because they respond to drastically changes in soil density, they can serve as bioindicators (Schmidt et al. 2013).

Aiming to develop an efficient sampling method for subterranean ants, researchers have been testing useful sampling techniques that can be replicated. Wong \& Guénard (2017) compiled the published research on the collecting methods of subterranean ants and classified them into three types: (1) subterranean baits, (2) soil sampling, and (3) direct sampling. Most of those publications (65\%) described the use of baits or other attractive items (Wong \& Guénard 2017), which favor the capture of predatory, generalist and mass-recruiting species (Longino 2000, Bestelmeyer et al. 2000, Schmidt \& Solar 2010, Wong \& Guénard 2017).

Amongst the methods classified as "soil sampling" is the extraction of monoliths (Delabie \& Fowler 1995, Fowler et al. 2000, Andersen \& Brault 2010, Wong \& Guénard 2017). The use of monoliths is promising because it enables us to determine the vertical stratification of species in the soil, among other advantages (see: Jacquemin et al. 2012, Jacquemin et al. 2016, Wong \& Guénard 2017). A widely used technique of monolith extraction in soil macrofauna studies was proposed by the Tropical Soil Biology and Fertility Program (TSBF) (Anderson \& Ingram 1993) and was standardized by the International Organization for Standardization (ISO) in the ISO norm 23611-5:2011. This technique, known as TSBF method, generates a substantial volume of ant specimens. It consists of the removal of soil monoliths that are $25 \times 25 \mathrm{~cm}$ and are taken 20 or $30 \mathrm{~cm}$ deep in the soil, followed by hand sorting extraction of the associated fauna (Bartz et al. 2014a, Rosa et al. 2015, ISO 2018).

Despite the massive volume of material that has been sampled using the TSBF method, published information on the ecological dynamics of the ants collected using it is insufficient. This is a function of the fact that Pedobiology studies (which employ the TSBF method with high frequencies) traditionally identify the collected material at high taxonomic levels (like Order, Family and Genera) and therefore do not give information on community composition and species ecology (see: Lourente et al. 2007, Klenk et al. 2009, Correia 2010, Lima et al. 2010, Rousseau et al 2010, Zagatto 2014, Benazzi et al. 2013, Pereira et al. 2012, Gutiérrez et al. 2017).

The present work characterizes subterranean ant assemblages in three subtropical regions of the Neotropics using two sampling methods: TSBF and epigaeic pitfall traps. In situ extraction methods, like monoliths, can help to detect ecological, behavioral and morphological patterns, since the specimens collected using these methods are not attracted from other strata (from the surface, for example). Furthermore,
TSBF is widely disseminated in studies that evaluate the impact of land use systems on the underground soil (micro, meso and macro fauna) (Lavelle et al 1997, Bartz et al 2014a, Rosa et al. 2015). In order to improve the scientific contribution of this study, we characterize the ant assemblages and compare the communities at the species level.

\section{Material and Methods}

\section{Study area}

The specimens came from three localities in the Southern Region of Brazil: (1) Ponta Grossa, Campos Gerais region, state of Paraná; (2) Western and (3) Plateau, regions of the state of Santa Catarina. In each region of Santa Catarina, the samples were collected from three municipalities: Xanxerê, Chapecó and São Miguel do Oeste in the West and Lages, Otacílio Costa and Campo Belo do Sul in the Plateau (Figure 1).

Ponta Grossa is in the Second Plateau of Paraná, about 120 kilometers from the state capital, Curitiba. According to Maack (2012), the region is characterized by open grasslands with Araucaria angustifolia (Bertol.) Kuntze 1898 patches, within the Atlantic Forest domain. Climate is type $\mathrm{Cfb}$ (climatic classification of Köppen-Geiger) -subtropical humid, without defined dry periods throughout the year and with the average temperature of the warmest month lower than $22{ }^{\circ} \mathrm{C}$. The sampled areas have mean elevation of $875 \mathrm{~m}$ and total annual rainfall between 1.300 and $1.800 \mathrm{~mm}$ throughout the year (IAPAR, 1978). Seven land use systems (LUSs) were sampled: (1) Integrated Crop-livestock (ICL); (2) Integrated Crop-livestock-forestry (ICLF); (3) Grazed native field (GN); (4) Eucalyptus sp. plantation (EP); (5) no-tillage (NT); (6) Campos Gerais (CG), and (7) Mixed Ombrophilous forest (F). The native areas, CG and F, were at Vila Velha State Park (2514'17" S 500'39" W). The land use systems ICL, ICLF, and GN were in the Model Farm of the Instituto Agronômico do Paraná (IAPAR) (25 5' $11^{\prime} \mathrm{S} 50^{\circ} 9$ ' 38' W) and the EU and NT systems were at the Experimental Field of the Empresa Brasileira de Pesquisas Agropecuárias (Embrapa) (2508'17” S 5004’47” W).

The western region of Santa Catarina is characterized by humid subtropical Cfa climate, with no defined dry season, and the average temperature during the warmest month is above $22{ }^{\circ} \mathrm{C}$ (Bartz et al. 2014a). The native forest in the Western region of the state is a transition between mixed ombrophilous and semi-deciduous forest (Bartz et al. 2014b). In the Plateau region the climate is classified as $\mathrm{Cfb}$ (as described for Ponta Grossa), and native grasslands with mixed ombrophilous forest patches predominate (Rosa 2013). The municipalities of each region of Santa Catarina were chosen based on their geographic characteristics, soil type, LUSs, and management history. Five LUSs were sampled in each of the three municipalities: (1) Native forest (FN); (2) Eucalyptus sp. plantation (EP); (3) Pasture (PA); (4) No-tillage (NT) and (5) Integrated Crop-livestock integration (ICL) (for details on the location and characterization of the areas see: Rosa 2013, Bartz et al. 2014a, b, Rosa et al. 2015).

All areas were part of a study of soil quality in different land use systems developed by the Embrapa Forestry (Colombo, PR), and Santa Catarina SisBiota Project, led by the Universidade Estadual de Santa Catarina (UDESC) at Chapecó. In these projects, soil fauna was evaluated as an indicator of soil quality in different LUS. 


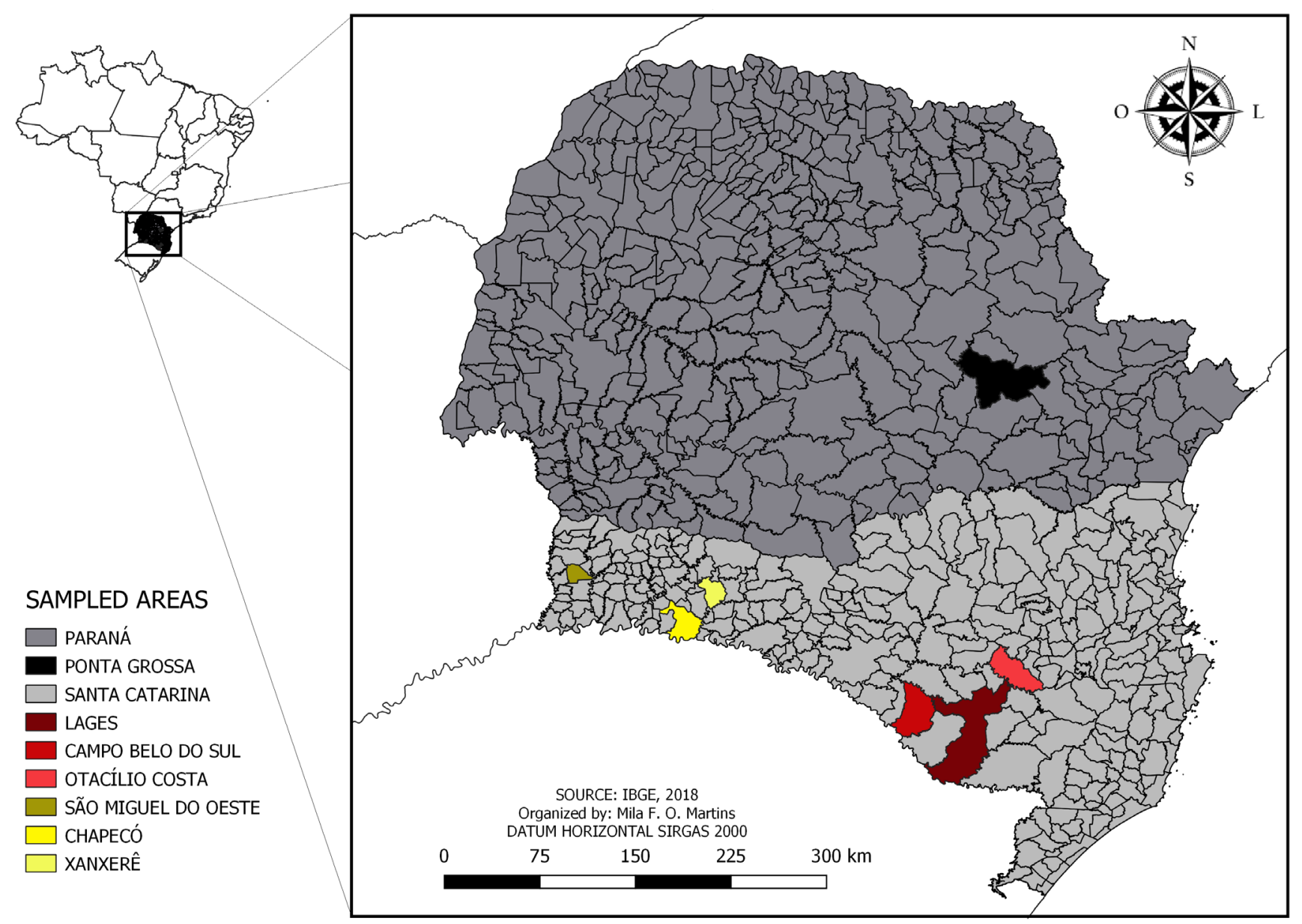

Figure 1. Map of the sampled areas.

\section{Sampling design}

The Tropical Soil Biology and Fertility Program organizes measures to monitor soil quality through the removal of monoliths in places where financial and technological resources are limited. The soil monoliths were $25 \times 25 \mathrm{~cm}$, and the depth at which they were collected was variable according to the extent of horizon A of the soil (Anderson \& Ingram 1993, ISO 2018). This method can be applied to any soil type. The area for the removal of monoliths is delimited by a metallic quadrangular structure, followed by the excavation and removal of soil layers. Each layer is packed separately, and the associated fauna can be extracted either in the field or in the laboratory, with or whitout assistance of a stereomicroscope (Anderson \& Ingram 1993). In the present study, all extractions were performed in the laboratory, without stereomicroscope. Our extractions, in Ponta Grossa, were made in layers up to $20 \mathrm{~cm}$, delimited in three strata: 0 - litter; I - 0 to $10 \mathrm{~cm}$ and II -10 to $20 \mathrm{~cm}$. In Santa Catarina, extractions were not stratified.

We used material from two different sample designs, according to the research group involved in the sampling. In Paraná, samples were collected on four different dates. In the areas of ICLF, ICL, NF, NT, and EU, samples were taken in October and November 2012 and in April and May 2013. In the Vila Velha State Park, samples were taken in September 2013 and in January 2014. All areas of Santa Catarina (West and Plateau) were sampled in the course of two seasons: winter (July and August 2011) and summer (December 2011 and January 2012).
In Paraná, for each area (seven LUSs) three plots of 50 x $100 \mathrm{~m}(0.5$ hectares) were delimited per LUS, within which five monoliths were extracted, separated by at least $200 \mathrm{~m}$. These samples were distributed in a central transect, 20 meters from the edge of the plot. A zigzag pattern was drawn with samples always at least $20 \mathrm{~m}$ apart and following the slope of the plot (Zagatto, 2014) (Figure 2). A total of 105 monoliths (seven LUSs $\mathrm{x}$ three plots $\mathrm{x}$ five monoliths) were collected, divided into the depth layers mentioned above ( 0 , I and II), totalling 315 sample units per season of the year (630 total samples).

In Santa Catarina, one area of each LUS (NF, EU, PA, ICL, and NT) was evaluated in each of the three municipalities per region (West and Plateau). Sampling grids, with nine points mounted in three lines of three points each, were sampled $20 \mathrm{~m}$ from the border and $30 \mathrm{~m}$ apart in each LUS, totaling one hectare. At each point a monolith was extracted and an epigaeic pitfall trap was installed (one meter distant from the monolith) (Figure 3). Each pitfall trap consisted of a $200 \mathrm{ml}$ capacity recipient, half filled with water and detergent, buried with the opening at ground level and left in the field for 72 hours. A total of 270 samples of TSBF and 270 of epigaeic pitfalls (three municipalities $x$ five LUSs $\mathrm{x}$ nine monoliths $\mathrm{x}$ two regions) were obtained per season (1080 total samples).

The ants from all areas were conditioned in $80 \%$ alcohol and sent to the Laboratório de Sistemática e Biologia de Formigas of the Universidade Federal do Paraná(UFPR). The material was processed 


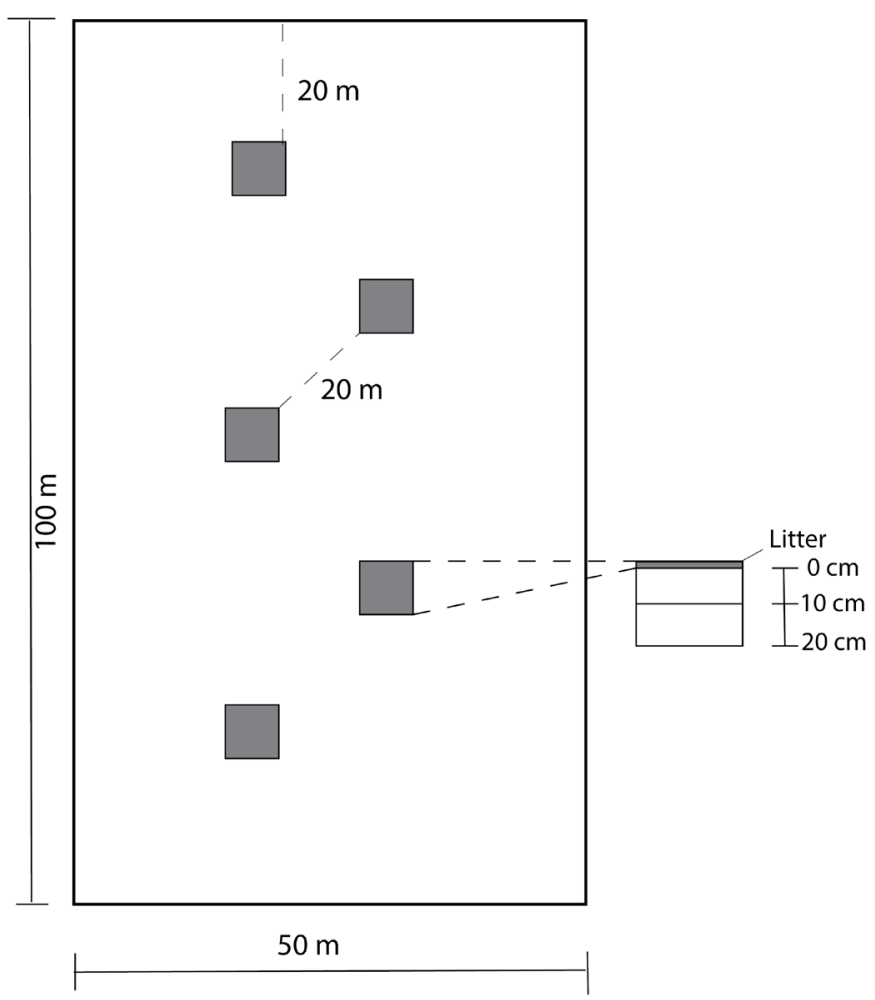

Figure 2. Schematic drawing of the sampling design in the state of Paraná. Adapted from Zagatto (2014).

and identified to the specific level whenever possible. The identifications were generally based on the Guide for the Ant Genera of Brazil (Baccaro et al., 2015). In addition, UFPR specialists (Alexandre C. Ferreira, Gabriela P. Camacho, John E. Latke, Mayron Escárraga, Rodrigo M. Feitosa, and Thiago S. R. da Silva) were consulted to confirm species identifications. Finally, whenever necessary, we performed comparisons with the material deposited in the Coleção Entomológica Padre Jesus Santiago Moure at UFPR (DZUP), where voucher material was deposited. The species that were not possible to name were identified as morphospecies.

\section{Data analysis}

Due to differences in the sampling design, the data were organized in two separate matrices that indicate presence and absence: one matrix with data from Paraná and the other with data from Santa Catarina. Since samples were taken at different seasons to increase the likelihood of finding different species, each species in a sampling unit was considered only once, regardless of the season when it was collected, as suggested by Brandão et al (2011). The observed richness (Sobs) obtained by each method and stratum were compared with the richness estimated by Jackniffe1 and Bootstrap. These estimators were chosen because they provide a satisfactory balance in the analyses, since they separately consider the effect of species occurring in a single sample (Jackniffe1) and of all species in the samples, without overweighting the presence of rare species (Bootstrap). To determine whether a species is epigaeic or hypogaeic, we used the exclusivity criterion: species collected only by the TSBF method or those present only in strata I and II were considered subterranean; conversely, the species collected only by pitfall traps or

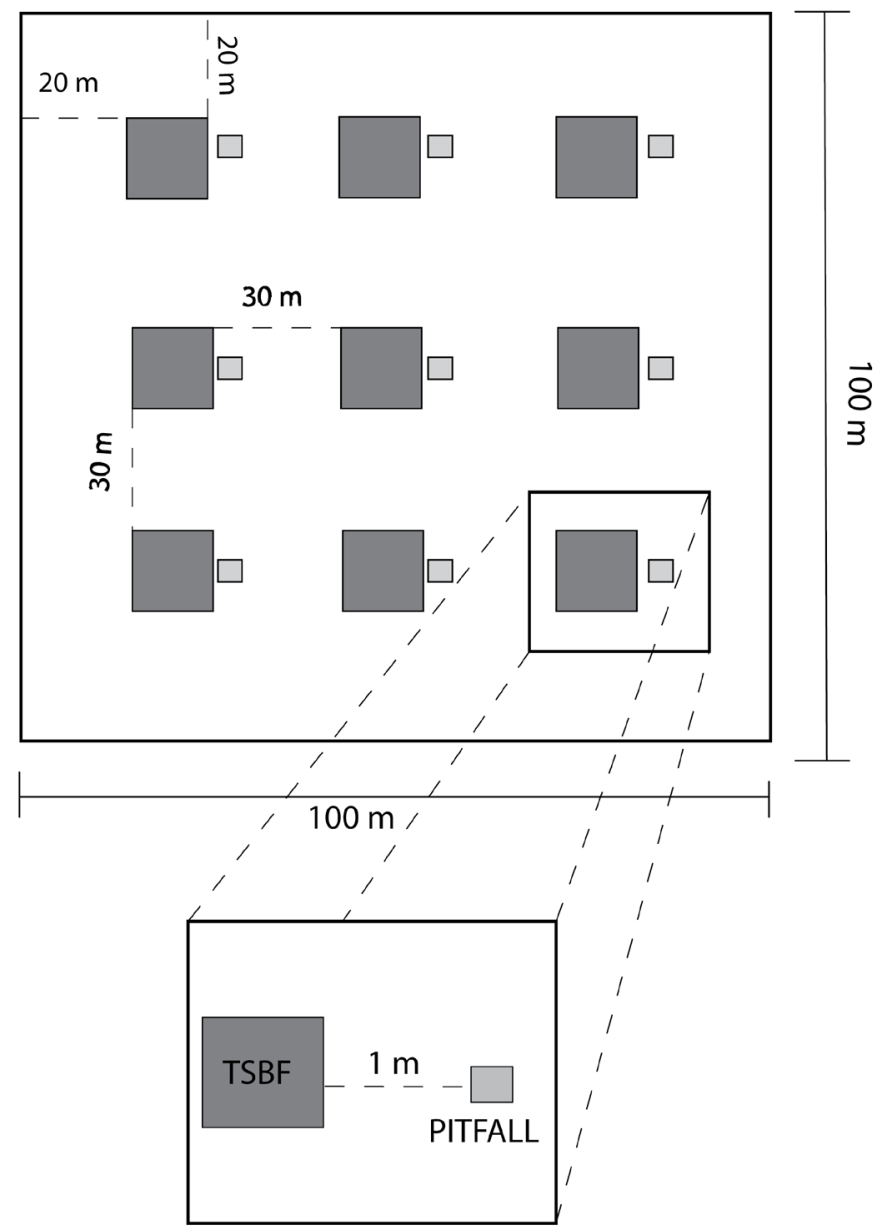

Figure 3. Schematic drawing of the sampling design in the state of Santa Catarina. Adapted from Bartz et al. (2014), and Rosa et al. (2015).

those present only in stratum 0 of the TSBF method were considered epigaeic. We used the samples from Paraná to evaluate the sampling efficiency of the TSBF method, since only these samples were stratified (layer separation by depth). The stratification allowed us to evaluate the presence of ants in the different strata, providing data not only on ant richness but also on aspects related to their biology (stratum of occurrence).

A generalized linear mixed model (GLMM) was used to verify if the presence of ants in strata was homogeneous (providing an equal probability of collection success between the layers). The model was elaborated from the proportion of ant occurrence in the plots (ranging from 0 to 5, in a half-hectare), with the strata as fixed variables and the LUSs as random variables. The model was compared by maximum likelihood and the data were evaluated for overdispersion, assuming a binomial distribution.

The analyses and graphs were performed in the $\mathrm{R}$ environment (version 3.1.3, R-CORE-TEAM 2016) with the vegan, car, MASS, iNEXT and lme4 packages. The map of the localities was generated using Program QGis v. 2.16.3 and the coordinates provided in Zagatto (2014) and Rosa (2013) and the shape files obtained from the Brazilian Institute of Geography and Statistics data base - IBGE (2016). The figures were drawn in Adobe Illustrator (version CC 2018). 


\section{Results}

\section{Richness and species frequency}

In total, 281 ant species were collected (considering all counties sampled and the different methods - epigaeic pitfall trap and TSBF). These species were distributed in 49 genera and 10 subfamilies (Table 1). The subfamilies with the greatest number of species were Myrmicinae, with 175 (61.4\% of the species collected); Formicinae, 39 (13.7\%); and Ponerinae, with 32 species (11.2\%). The richest genera were Pheidole, with 98 species (34\%), Solenopsis and Hypoponera with 22 species (7.7\%) each.
In Santa Catarina, 238 species were collected by both methods (epigaeic pitfall and TSBF). Pitfalls collected 202 species and generated 1,585 records of occurrence, while the TSBF collected 149 species, generating 769 records (Table 2). From the total, 89 species were collected only in pitfall $(51.7 \%$ of them composed by the genus Pheidole) and 36 only in the TSBF (Table 1). The three most frequent species in the pitfalls were Pachycondyla striata Smith, 1858, Pheidole subarmata Mayr, 1884 and Linepithema micans (Forel, 1908), with 109, 97 and 71 occurrences, respectively. In TSBF the species with the greatest number of records were Hypoponera sp. 1, Hypoponera sp. 8 and Pheidole subarmata, with 60, 57 and 42 records, respectively.

Table 1. Species list by region (Campos Gerais, West and Plateau) and municipality. PG - Ponta Grossa, SMO - São Miguel do Oeste, CHP - Chapecó, XAN - Xanxerê, LGS - Lages, OTC - Otacílio Costa, and CBS - Campo Belo do Sul). The species sampled exclusively by a method (in Santa Catarina) are indicated by symbols: - TSBF, - Pitfall; and the exclusive species per stratum are indicated with superscript numbers: ${ }^{0}-$ litter, ${ }^{\mathrm{I}}$ - stratum I $(0-10 \mathrm{~cm})$, and ${ }^{\mathrm{II}}$ - stratum II $(10-20 \mathrm{~cm})$.

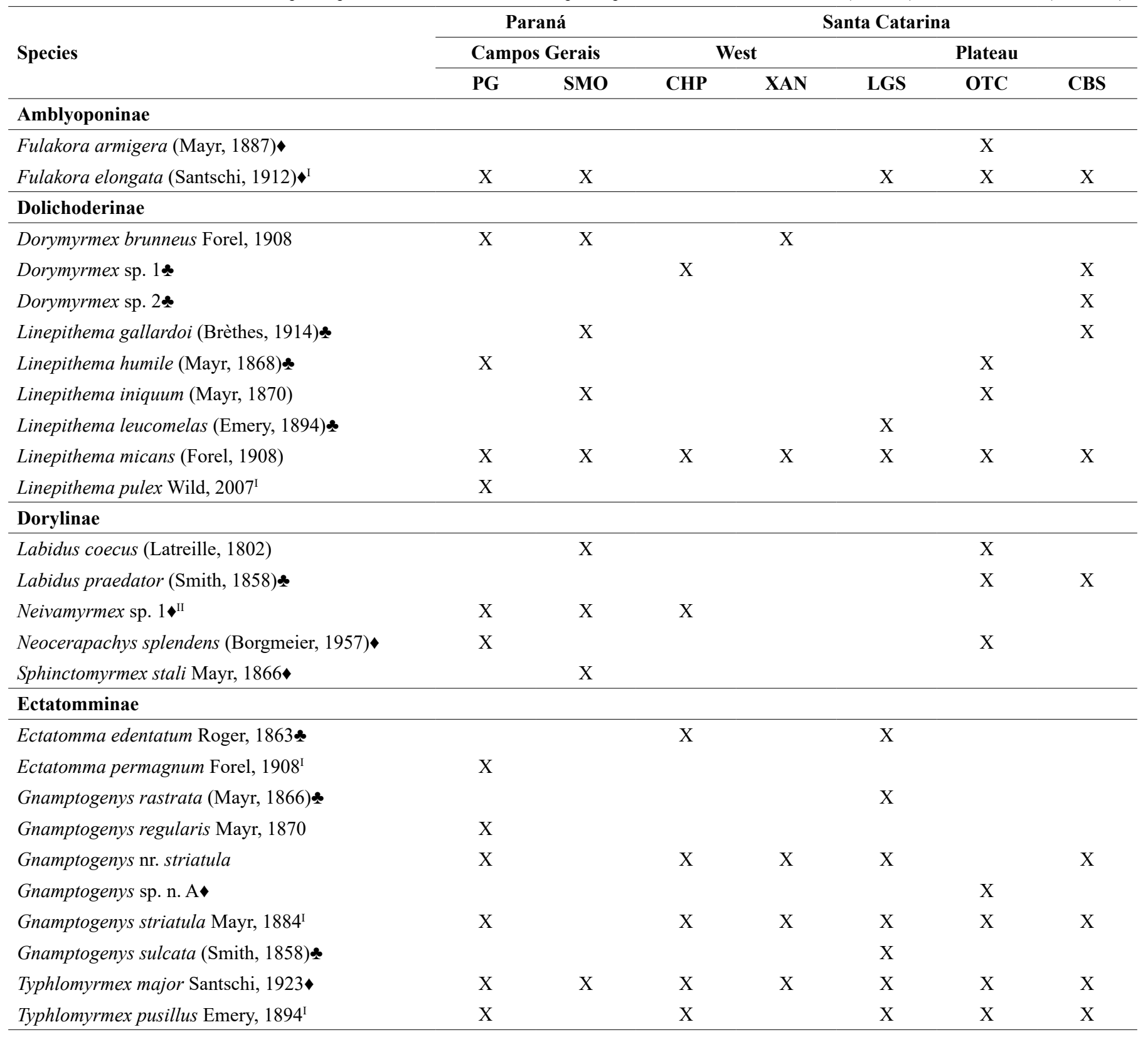


Martins, M.F.O. et al.

Continuation Table 1.

\begin{tabular}{|c|c|c|c|c|c|c|c|}
\hline \multirow{3}{*}{ Species } & \multirow{2}{*}{\multicolumn{2}{|c|}{$\begin{array}{c}\text { Paraná } \\
\text { Campos Gerais }\end{array}$}} & \multicolumn{5}{|c|}{ Santa Catarina } \\
\hline & & & \multicolumn{2}{|c|}{ West } & \multicolumn{3}{|c|}{ Plateau } \\
\hline & PG & SMO & CHP & XAN & LGS & OTC & CBS \\
\hline \multicolumn{8}{|l|}{ Formicinae } \\
\hline Acropyga decedens (Mayr, 1887) & & & & & $\mathrm{X}$ & & \\
\hline Acropyga goeldii Forel, 1893 & $\mathrm{X}$ & & & $\mathrm{X}$ & $\mathrm{x}$ & $\mathrm{X}$ & \\
\hline Brachymyrmex coactus Mayr, $1887^{1}$ & $\mathrm{X}$ & & & & & & \\
\hline Brachymyrmex pilipes Mayr, 1887 & $\mathrm{X}$ & & $\mathrm{x}$ & & & & \\
\hline Brachymyrmex pilipes gr. sp. $1 \downarrow$ & & $\mathrm{X}$ & & & & & \\
\hline Brachymyrmex sp. 1 & $\mathrm{X}$ & $\mathrm{X}$ & $\mathrm{X}$ & $\mathrm{X}$ & $\mathrm{X}$ & $\mathrm{X}$ & \\
\hline Brachymyrmex sp. 2 & $\mathrm{X}$ & $\mathrm{X}$ & $\mathrm{X}$ & $\mathrm{X}$ & $\mathrm{X}$ & $\mathrm{X}$ & $\mathrm{X}$ \\
\hline Brachymyrmex sp. 3 & $\mathrm{X}$ & $\mathrm{X}$ & $\mathrm{X}$ & $\mathrm{X}$ & $\mathrm{X}$ & $\mathrm{X}$ & $\mathrm{X}$ \\
\hline Brachymyrmex sp. 4 & $\mathrm{X}$ & & & & & & \\
\hline Brachymyrmex sp. 5 & $\mathrm{X}$ & $\mathrm{X}$ & $\mathrm{X}$ & $\mathrm{X}$ & & $\mathrm{X}$ & \\
\hline Brachymyrmex sp. 6 & $\mathrm{X}$ & $\mathrm{X}$ & & $\mathrm{X}$ & & $\mathrm{X}$ & \\
\hline Brachymyrmex sp. 7 & $\mathrm{X}$ & & $\mathrm{X}$ & $\mathrm{X}$ & $\mathrm{X}$ & $\mathrm{X}$ & $\mathrm{X}$ \\
\hline Brachymyrmex sp. & & & & & $\mathrm{X}$ & & \\
\hline Brachymyrmex sp. 9 & & $\mathrm{X}$ & & & & $\mathrm{X}$ & \\
\hline Brachymyrmex sp. 10 & & $\mathrm{X}$ & & $\mathrm{X}$ & & $\mathrm{X}$ & \\
\hline Camponotus balzani Emery, 1894 & & & & & $\mathrm{X}$ & $\mathrm{X}$ & $\mathrm{x}$ \\
\hline Camponotus blandus (Smith, 1858) & & & & & $\mathrm{x}$ & $\mathrm{X}$ & $\mathrm{X}$ \\
\hline Camponotus brasiliensis Mayr, $1862^{1}$ & $\mathrm{X}$ & & & & & & \\
\hline Camponotus crassus Mayr, 1862 & $\mathrm{X}$ & $\mathrm{X}$ & $\mathrm{X}$ & & & $\mathrm{X}$ & $\mathrm{X}$ \\
\hline Camponotus melanoticus Emery, 1894 & $\mathrm{x}$ & $\mathrm{x}$ & $\mathrm{X}$ & & $\mathrm{X}$ & & $\mathrm{X}$ \\
\hline Camponotus novogranadensis Mayr, 1870 & $\mathrm{X}$ & & $\mathrm{x}$ & $\mathrm{X}$ & & & \\
\hline Camponotus nr. cingulatus & & & & & $\mathrm{X}$ & & $\mathrm{X}$ \\
\hline Camponotus punctulatus Mayr, 1868 & & & & & $\mathrm{X}$ & $\mathrm{X}$ & $\mathrm{X}$ \\
\hline Camponotus renggeri Emery, 1894"II & $\mathrm{X}$ & & $\mathrm{X}$ & $\mathrm{X}$ & & & $\mathrm{X}$ \\
\hline Camponotus rufipes (Fabricius, 1775) & & $\mathrm{X}$ & $\mathrm{x}$ & $\mathrm{X}$ & & $\mathrm{X}$ & $\mathrm{X}$ \\
\hline Camponotus sexguttatus (Fabricius, 1793)"I & $\mathrm{x}$ & & & & & & \\
\hline Camponotus zenon Forel, 1912 & & & $\mathrm{X}$ & $\mathrm{X}$ & & & $\mathrm{X}$ \\
\hline Camponotus sp. $1^{\mathrm{I}}$ & $\mathrm{X}$ & & & & & & \\
\hline Camponotus sp. 2 & $\mathrm{X}$ & & & & & & \\
\hline Camponotus sp. 4 & & & & & & & $\mathrm{X}$ \\
\hline Camponotus sp. 5 & & & & & & & $\mathrm{X}$ \\
\hline Camponotus sp. 6 & & & & $\mathrm{X}$ & & & \\
\hline Myrmelachista catharinae Mayr, 1887 & & & & $\mathrm{X}$ & & & \\
\hline Myrmelachista nodigera Mayr, 1887 & & & & & & $\mathrm{X}$ & \\
\hline Myrmelachista gallicola Mayr, $1887^{1}$ & $\mathrm{X}$ & & & & & & \\
\hline Nylanderia fulva $($ Mayr, 1862) & $\mathrm{X}$ & $\mathrm{X}$ & $\mathrm{X}$ & $\mathrm{X}$ & & & \\
\hline Nylanderia sp. 1 & $\mathrm{X}$ & $\mathrm{X}$ & $\mathrm{x}$ & $\mathrm{X}$ & & $\mathrm{X}$ & \\
\hline Nylanderia sp. 2 & & & $\mathrm{x}$ & $\mathrm{X}$ & $\mathrm{X}$ & & \\
\hline Nylanderia sp. 3 & & $\mathrm{X}$ & & & & & \\
\hline
\end{tabular}


Subterranean ants of southern Brazil

Continuation Table 1.

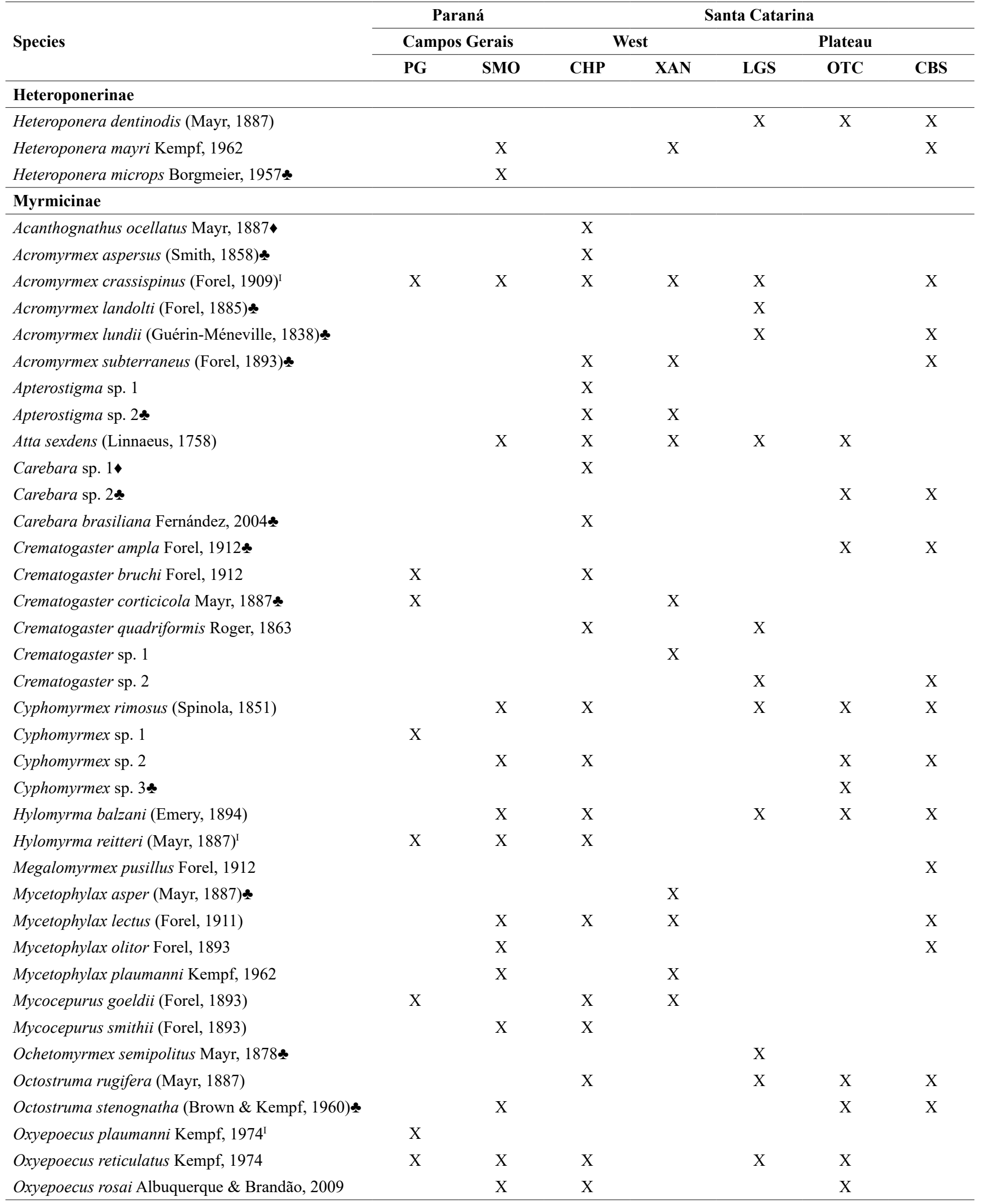


Martins, M.F.O. et al.

Continuation Table 1.

\begin{tabular}{|c|c|c|c|c|c|c|c|}
\hline \multirow{3}{*}{ Species } & \multirow{2}{*}{\multicolumn{2}{|c|}{$\begin{array}{c}\text { Paraná } \\
\text { Campos Gerais }\end{array}$}} & \multicolumn{5}{|c|}{ Santa Catarina } \\
\hline & & & \multicolumn{2}{|c|}{ West } & \multicolumn{3}{|c|}{ Plateau } \\
\hline & PG & SMO & CHP & XAN & LGS & OTC & CBS \\
\hline Pheidole aberrans Mayr, 1868 & $\mathrm{X}$ & $\mathrm{X}$ & $\mathrm{X}$ & $\mathrm{X}$ & $\mathrm{X}$ & $\mathrm{X}$ & $\mathrm{X}$ \\
\hline Pheidole acutidens (Santschi, 1922) & & & & & & $\mathrm{X}$ & \\
\hline Pheidole breviseta Santschi, 1919 & & $\mathrm{X}$ & $\mathrm{X}$ & $\mathrm{X}$ & $\mathrm{X}$ & $\mathrm{X}$ & $\mathrm{X}$ \\
\hline Pheidole cavifrons Emery, 1906 & $\mathrm{X}$ & & & & $\mathrm{X}$ & $\mathrm{X}$ & $\mathrm{X}$ \\
\hline Pheidole fallax gr. sp. $1^{0}$ & $\mathrm{X}$ & & & & & & \\
\hline Pheidole gertrudae Forel, $1886^{0}$ & $\mathrm{X}$ & $\mathrm{X}$ & $\mathrm{X}$ & $\mathrm{X}$ & & & \\
\hline Pheidole heyeri Forel, 1899 & & $\mathrm{X}$ & $\mathrm{X}$ & $\mathrm{X}$ & $\mathrm{X}$ & $\mathrm{X}$ & $X$ \\
\hline Pheidole oxyops Forel, 1908 & $\mathrm{x}$ & & & & & & \\
\hline Pheidole nr. rosula & & & & & $\mathrm{X}$ & $\mathrm{X}$ & $\mathrm{X}$ \\
\hline Pheidole radoszkowskii Mayr, $1884^{1}$ & $\mathrm{X}$ & & & & & & \\
\hline Pheidole rosae Forel, 1901 & $\mathrm{X}$ & & & & & & \\
\hline Pheidole subarmata Mayr, 1884 & $\mathrm{X}$ & $\mathrm{X}$ & $\mathrm{X}$ & $\mathrm{X}$ & $\mathrm{X}$ & $\mathrm{X}$ & $\mathrm{X}$ \\
\hline Pheidole transversostriata Mayr, 1887 & & $\mathrm{X}$ & & & & & \\
\hline Pheidole triconstricta Forel, 1886 & & $\mathrm{X}$ & $\mathrm{X}$ & $\mathrm{X}$ & & & \\
\hline Pheidole vafra Santschi, 1923 & $\mathrm{X}$ & & & & & & \\
\hline Pheidole sp. n. A & $\mathrm{X}$ & & & & & & \\
\hline Pheidole sp. n. $\mathrm{I}^{\mathrm{I}}$ & $\mathrm{X}$ & & & & & & \\
\hline Pheidole sp. $\mathrm{n} . \mathrm{N}^{\mathrm{I}}$ & $\mathrm{X}$ & & & & & & \\
\hline Pheidole sp. 1 & $\mathrm{X}$ & & & & & & \\
\hline Pheidole sp. $2{ }^{I}$ & $\mathrm{X}$ & & $\mathrm{X}$ & & & & \\
\hline Pheidole sp. $3^{\mathrm{I}}$ & $\mathrm{X}$ & & & & & & \\
\hline Pheidole sp. 4 & $\mathrm{X}$ & & & & & & \\
\hline Pheidole sp. $5^{1}$ & $\mathrm{x}$ & & & & & & \\
\hline Pheidole sp. 6 & $\mathrm{X}$ & & & & & & \\
\hline Pheidole sp. 7 & $\mathrm{X}$ & & & & & & \\
\hline Pheidole sp. 8 & $\mathrm{X}$ & & & & & & \\
\hline Pheidole sp. 9 & $\mathrm{X}$ & & & & & & \\
\hline Pheidole sp. 10 & $\mathrm{X}$ & & & & & & \\
\hline Pheidole sp. 11 & & $\mathrm{X}$ & & & $\mathrm{X}$ & $\mathrm{X}$ & $\mathrm{X}$ \\
\hline Pheidole sp. 12 & & $\mathrm{X}$ & & & & & $\mathrm{X}$ \\
\hline Pheidole sp. 13 & & & & & $\mathrm{X}$ & $\mathrm{X}$ & \\
\hline Pheidole sp. 14 & & $\mathrm{X}$ & & & $\mathrm{X}$ & $\mathrm{X}$ & $\mathrm{X}$ \\
\hline Pheidole sp. 15 & & $\mathrm{x}$ & $\mathrm{X}$ & & $\mathrm{X}$ & & $\mathrm{X}$ \\
\hline Pheidole sp. 16 & & $\mathrm{x}$ & $\mathrm{x}$ & $\mathrm{X}$ & & $\mathrm{X}$ & $\mathrm{X}$ \\
\hline Pheidole sp. 17. & & $\mathrm{X}$ & & $\mathrm{X}$ & $\mathrm{X}$ & & \\
\hline Pheidole sp. 18 & & & & & $\mathrm{X}$ & $\mathrm{X}$ & \\
\hline Pheidole sp. 19 & & $\mathrm{X}$ & $\mathrm{X}$ & $\mathrm{X}$ & $\mathrm{X}$ & $\mathrm{X}$ & $\mathrm{X}$ \\
\hline Pheidole sp. 20 & & & $\mathrm{X}$ & $\mathrm{X}$ & $\mathrm{X}$ & $\mathrm{X}$ & $\mathrm{X}$ \\
\hline Pheidole sp. 21 & & $\mathrm{X}$ & & & $\mathrm{X}$ & $\mathrm{X}$ & \\
\hline Pheidole sp. 22 & & & & & & $\mathrm{X}$ & \\
\hline Pheidole sp. 23 & & & & & $\mathrm{X}$ & & \\
\hline Pheidole sp. 24 & & $\mathrm{X}$ & & $\mathrm{X}$ & & $\mathrm{X}$ & \\
\hline Pheidole sp. 25 & & $\mathrm{X}$ & & & & & \\
\hline
\end{tabular}


Continuation Table 1.

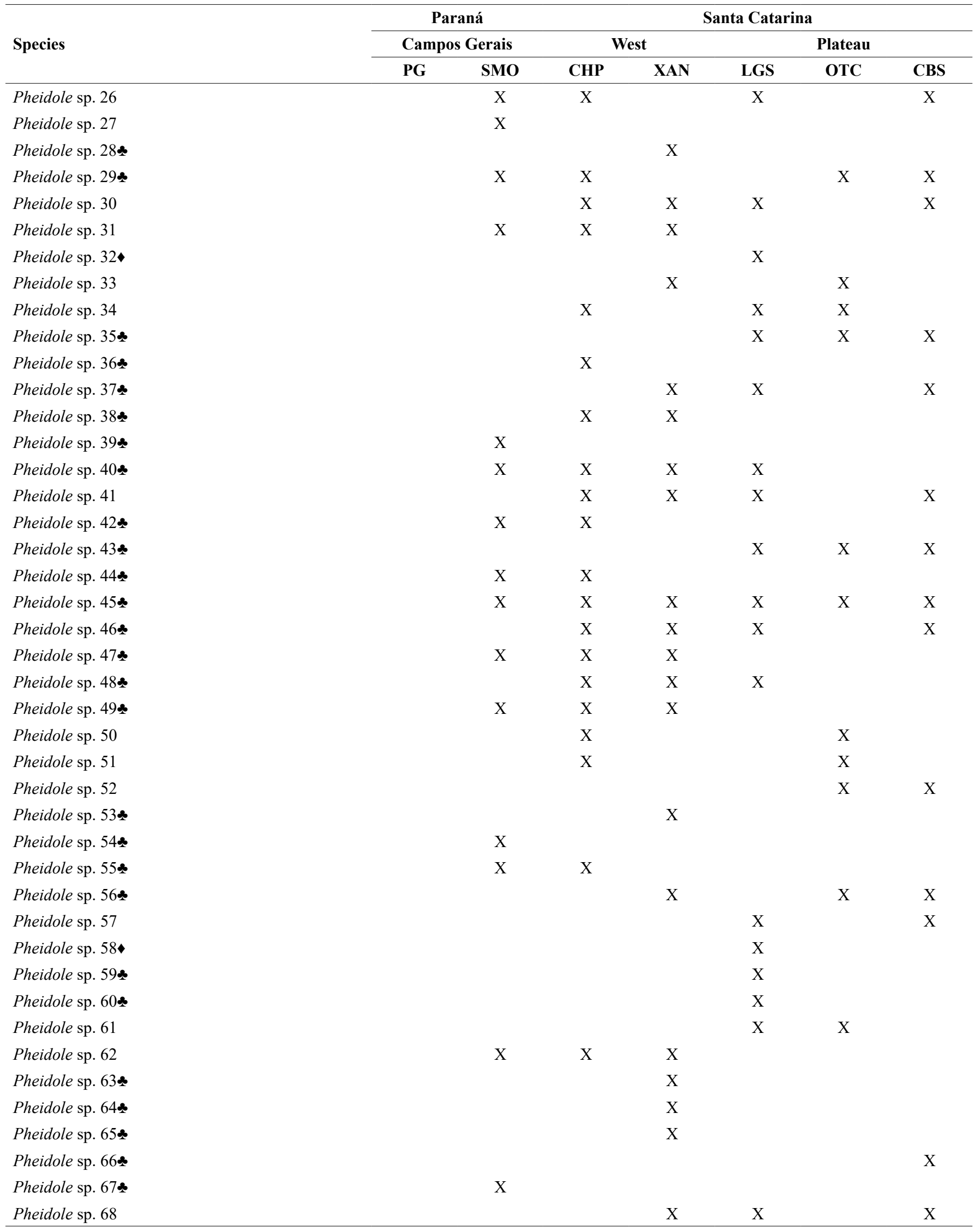


Martins, M.F.O. et al.

Continuation Table 1.

\begin{tabular}{|c|c|c|c|c|c|c|c|}
\hline \multirow{3}{*}{ Species } & \multirow{2}{*}{\multicolumn{2}{|c|}{$\begin{array}{c}\text { Paraná } \\
\text { Campos Gerais }\end{array}$}} & \multicolumn{5}{|c|}{ Santa Catarina } \\
\hline & & & \multicolumn{2}{|c|}{ West } & \multicolumn{3}{|c|}{ Plateau } \\
\hline & PG & SMO & CHP & XAN & LGS & OTC & CBS \\
\hline Pheidole sp. 69 & & & & & & $\mathrm{X}$ & \\
\hline Pheidole sp. 70 & & & & & $\mathrm{X}$ & & \\
\hline Pheidole sp. 71 & & $\mathrm{X}$ & & & & & \\
\hline Pheidole sp. 72 & & & & & $\mathrm{X}$ & $\mathrm{X}$ & $\mathrm{X}$ \\
\hline Pheidole sp. 73 & & & & & $\mathrm{x}$ & & $\mathrm{X}$ \\
\hline Pheidole sp. 74 & & & & & & $\mathrm{X}$ & \\
\hline Pheidole sp. 75 & & & & & $\mathrm{X}$ & & $\mathrm{X}$ \\
\hline Pheidole sp. 76 & & & & $\mathrm{X}$ & & $\mathrm{X}$ & $\mathrm{X}$ \\
\hline Pheidole sp. 77 & & & & $\mathrm{X}$ & $\mathrm{X}$ & $\mathrm{x}$ & \\
\hline Pheidole sp. 78 & & & & & & & $\mathrm{X}$ \\
\hline Pheidole sp. 79 & & & & $\mathrm{X}$ & & & \\
\hline Pheidole sp. 80 & & $\mathrm{X}$ & & & & & \\
\hline Pogonomyrmex naegelli Emery, 1878 & $\mathrm{X}$ & & $\mathrm{X}$ & & $\mathrm{X}$ & $\mathrm{X}$ & \\
\hline Rogeria bruchi Santschi, 1922 & & & & & $\mathrm{X}$ & & \\
\hline Solenopsis gr. geminata sp. $1^{\mathrm{I}}$ & $\mathrm{X}$ & & & & & & \\
\hline Solenopsis gr. geminata sp. 2 & & $\mathrm{X}$ & $\mathrm{X}$ & $\mathrm{X}$ & $\mathrm{X}$ & $\mathrm{X}$ & $\mathrm{X}$ \\
\hline Solenopsis gr. geminata sp. 3 & & & $\mathrm{X}$ & $\mathrm{X}$ & & & \\
\hline Solenopsis invicta Buren, 1972 & $\mathrm{X}$ & $\mathrm{X}$ & $\mathrm{X}$ & $\mathrm{X}$ & $\mathrm{X}$ & $\mathrm{X}$ & $\mathrm{X}$ \\
\hline Solenopsis sp. 1 & $\mathrm{X}$ & $\mathrm{x}$ & & $\mathrm{X}$ & & & \\
\hline Solenopsis sp. 2 & $\mathrm{X}$ & $\mathrm{x}$ & & & & $\mathrm{X}$ & \\
\hline Solenopsis sp. $3^{\mathrm{I}}$ & $\mathrm{X}$ & & & $\mathrm{X}$ & & $\mathrm{X}$ & $\mathrm{X}$ \\
\hline Solenopsis sp. 4 & $\mathrm{X}$ & & & & & & \\
\hline Solenopsis sp. 5 & $\mathrm{X}$ & & & & & & \\
\hline Solenopsis sp. $6^{\mathrm{II}}$ & $\mathrm{X}$ & & & & & & \\
\hline Solenopsis sp. 7 & $\mathrm{X}$ & & & & & $\mathrm{X}$ & \\
\hline Solenopsis sp. $8^{\mathrm{I}}$ & $\mathrm{X}$ & & & & & & \\
\hline Solenopsis sp. 9 & $\mathrm{X}$ & $\mathrm{X}$ & $\mathrm{X}$ & $\mathrm{X}$ & $\mathrm{X}$ & $\mathrm{X}$ & $\mathrm{X}$ \\
\hline Solenopsis sp. 10 & & $\mathrm{X}$ & $\mathrm{X}$ & $\mathrm{X}$ & $\mathrm{x}$ & $\mathrm{X}$ & $\mathrm{X}$ \\
\hline Solenopsis sp. 11 & & & $\mathrm{X}$ & $\mathrm{X}$ & $\mathrm{X}$ & & $\mathrm{X}$ \\
\hline Solenopsis sp. 12 & & $\mathrm{X}$ & $\mathrm{X}$ & $\mathrm{X}$ & $\mathrm{X}$ & $\mathrm{X}$ & $\mathrm{X}$ \\
\hline Solenopsis sp. 13 & & $\mathrm{X}$ & $\mathrm{x}$ & $\mathrm{x}$ & $\mathrm{X}$ & $\mathrm{X}$ & $\mathrm{X}$ \\
\hline Solenopsis sp. 14 & & $\mathrm{X}$ & $\mathrm{x}$ & & $\mathrm{X}$ & $\mathrm{X}$ & \\
\hline Solenopsis sp. 15 & & $\mathrm{X}$ & $\mathrm{x}$ & $\mathrm{X}$ & $\mathrm{X}$ & $\mathrm{X}$ & $\mathrm{X}$ \\
\hline Solenopsis sp. 16 & & $\mathrm{X}$ & & & & & $\mathrm{X}$ \\
\hline Solenopsis sp. 17 & & & & & $\mathrm{X}$ & $\mathrm{X}$ & \\
\hline Solenopsis sp. 18 & & & & $\mathrm{X}$ & & & \\
\hline Strumigenys appretiata (Borgmeier, 1954) & & & & & $\mathrm{X}$ & & $\mathrm{X}$ \\
\hline Strumigenys denticulata Mayr, $1887 \downarrow$ & & & $\mathrm{X}$ & & & & \\
\hline Strumigenys eggersi Emery, 1890 & & $\mathrm{X}$ & $\mathrm{x}$ & & & & \\
\hline Strumigenys louisianae Roger, $1863^{1}$ & $\mathrm{X}$ & & & & & & \\
\hline Strumigenys oglobini Santschi, 1936 & & $\mathrm{X}$ & & & & & \\
\hline Strumigenys nr. epinotalis ${ }^{\mathrm{I}}$ & $\mathrm{X}$ & & & & & & \\
\hline
\end{tabular}


Continuation Table 1.

\begin{tabular}{|c|c|c|c|c|c|c|c|}
\hline \multirow{3}{*}{ Species } & \multirow{2}{*}{\multicolumn{2}{|c|}{$\begin{array}{c}\text { Paraná } \\
\text { Campos Gerais }\end{array}$}} & \multicolumn{5}{|c|}{ Santa Catarina } \\
\hline & & & \multicolumn{2}{|c|}{ West } & \multicolumn{3}{|c|}{ Plateau } \\
\hline & PG & SMO & CHP & XAN & LGS & OTC & CBS \\
\hline Strumigenys nr. louisianae sp. 1 & & & & & $\mathrm{X}$ & & \\
\hline Strumigenys nr. louisianae sp. 2 & & & $\mathrm{X}$ & $\mathrm{X}$ & $\mathrm{X}$ & $\mathrm{X}$ & \\
\hline Strumigenys nr. louisianae sp. 3 & & & & & $\mathrm{X}$ & & \\
\hline Mycetomoellerius holmgreni Wheeler, 1925 & & & & & $\mathrm{X}$ & $\mathrm{X}$ & $\mathrm{X}$ \\
\hline Mycetomoellerius sp. 1 & & & & & $\mathrm{X}$ & $\mathrm{X}$ & $\mathrm{X}$ \\
\hline Mycetomoellerius sp. 2 & & & & & $\mathrm{X}$ & & \\
\hline Tranopelta gilva Mayr, 1866 & $\mathrm{X}$ & & & & $\mathrm{X}$ & & \\
\hline Wasmannia affinis Santschi, 1929 & $\mathrm{X}$ & $\mathrm{X}$ & $\mathrm{X}$ & & $\mathrm{X}$ & $\mathrm{X}$ & \\
\hline Wasmannia auropunctata (Roger, 1863) & & $\mathrm{X}$ & $\mathrm{X}$ & $\mathrm{X}$ & $\mathrm{X}$ & $\mathrm{X}$ & $\mathrm{X}$ \\
\hline Wasmannia sulcaticeps Emery, 1894 & & $\mathrm{X}$ & $\mathrm{X}$ & & $\mathrm{X}$ & $\mathrm{X}$ & \\
\hline \multicolumn{8}{|l|}{ Ponerinae } \\
\hline Centromyrmex brachycola (Roger, 1861) & $\mathrm{X}$ & & & & & & \\
\hline Hypoponera foreli (Mayr, 1887) & $\mathrm{X}$ & $\mathrm{X}$ & $\mathrm{X}$ & $\mathrm{X}$ & $\mathrm{X}$ & $\mathrm{X}$ & $\mathrm{X}$ \\
\hline Hypoponera opaciceps (Mayr, 1887) & & $\mathrm{X}$ & $\mathrm{X}$ & $\mathrm{X}$ & $\mathrm{X}$ & $\mathrm{X}$ & $\mathrm{X}$ \\
\hline Hypoponera sp. 1 & $\mathrm{X}$ & $\mathrm{X}$ & $\mathrm{X}$ & $\mathrm{X}$ & $\mathrm{X}$ & $\mathrm{X}$ & $\mathrm{X}$ \\
\hline Hypoponera sp. 2 & $\mathrm{X}$ & $\mathrm{X}$ & $\mathrm{X}$ & $\mathrm{X}$ & & & $\mathrm{X}$ \\
\hline Hypoponera sp. 3 & $\mathrm{X}$ & & & & & & \\
\hline Hypoponera sp. 4 & $\mathrm{X}$ & & & & & & \\
\hline Hypoponera sp. 5 & $\mathrm{X}$ & & $\mathrm{X}$ & & $\mathrm{X}$ & $\mathrm{X}$ & \\
\hline Hypoponera sp. 6 I & $\mathrm{X}$ & $\mathrm{X}$ & & $\mathrm{X}$ & $\mathrm{X}$ & $\mathrm{X}$ & $\mathrm{X}$ \\
\hline Hypoponera sp. 7 & $\mathrm{X}$ & & & & & & \\
\hline Hypoponera sp. 8 & $\mathrm{X}$ & $\mathrm{X}$ & $\mathrm{X}$ & $\mathrm{X}$ & $\mathrm{X}$ & $\mathrm{X}$ & $\mathrm{X}$ \\
\hline Hypoponera sp. $9^{0}$ & $\mathrm{X}$ & & & & & & \\
\hline Hypoponera sp. $10^{1}$ & $\mathrm{X}$ & & & & $\mathrm{X}$ & $\mathrm{X}$ & $\mathrm{X}$ \\
\hline Hypoponera sp. 11 & $\mathrm{X}$ & & $\mathrm{X}$ & $\mathrm{X}$ & $\mathrm{X}$ & $\mathrm{X}$ & $\mathrm{X}$ \\
\hline Hypoponera sp. $12^{\mathrm{II}}$ & $\mathrm{X}$ & & & & & & \\
\hline Hypoponera sp. 13 & & $\mathrm{X}$ & & & $\mathrm{X}$ & $\mathrm{X}$ & $\mathrm{X}$ \\
\hline Hypoponera sp. 14 & & & $\mathrm{X}$ & & & $\mathrm{X}$ & \\
\hline Hypoponera sp. 15 & & & & & & $\mathrm{X}$ & $\mathrm{X}$ \\
\hline Hypoponera sp. 16 & & $\mathrm{X}$ & $\mathrm{X}$ & & $\mathrm{X}$ & $\mathrm{X}$ & $\mathrm{X}$ \\
\hline Hypoponera sp. 17 & & & $\mathrm{X}$ & $\mathrm{X}$ & & & \\
\hline Hypoponera sp. 18 & & & & & $\mathrm{X}$ & & \\
\hline Hypoponera sp. 19 & & & & & & $\mathrm{X}$ & \\
\hline Hypoponera sp. 21 & & & & & & $\mathrm{X}$ & \\
\hline Hypoponera sp. 22 & & & & $\mathrm{X}$ & & & \\
\hline Hypoponera sp. 23 & $\mathrm{X}$ & $\mathrm{X}$ & & & & & \\
\hline Neoponera bucki (Borgmeier, 1927) & & & & & $\mathrm{X}$ & & $\mathrm{X}$ \\
\hline Odontomachus chelifer (Latreille, 1802) & & $\mathrm{X}$ & $\mathrm{X}$ & $\mathrm{X}$ & & & \\
\hline Pachycondyla harpax (Fabricius, 1804) & & $\mathrm{X}$ & $\mathrm{X}$ & $\mathrm{X}$ & & & $\mathrm{X}$ \\
\hline Pachycondyla striata Smith, 1858 & & $\mathrm{X}$ & $\mathrm{X}$ & $\mathrm{X}$ & $\mathrm{X}$ & $\mathrm{X}$ & $\mathrm{X}$ \\
\hline Rasopone ferruginea (Smith, 1858) & & & & $\mathrm{X}$ & & & \\
\hline Simopelta curvata (Mayr, 1887) & & $\mathrm{X}$ & $\mathrm{X}$ & & & & \\
\hline
\end{tabular}




\begin{tabular}{|c|c|c|c|c|c|c|c|}
\hline \multirow{3}{*}{ Species } & \multirow{2}{*}{\multicolumn{2}{|c|}{$\begin{array}{c}\text { Paraná } \\
\text { Campos Gerais }\end{array}$}} & \multicolumn{5}{|c|}{ Santa Catarina } \\
\hline & & & \multicolumn{2}{|c|}{ West } & \multicolumn{3}{|c|}{ Plateau } \\
\hline & PG & SMO & CHP & XAN & LGS & OTC & CBS \\
\hline Discothyrea sexarticulata Borgmeier, $1954^{0}$ & $\mathrm{X}$ & & & & & & \\
\hline Proceratium brasiliense Borgmeier, 1959 & & & & & \multicolumn{3}{|c|}{$\mathrm{X}$} \\
\hline Pseudomyrmex gracilis (Fabricius, 1804) ${ }^{\mathrm{I}}$ & $\mathrm{X}$ & & & & & & \\
\hline Pseudomyrmex longus (Forel, 1912) ${ }^{\mathrm{I}}$ & $\mathrm{X}$ & & & & & & \\
\hline Pseudomyrmex termitarius (Smith, 1877) & & & $\mathrm{X}$ & & & & \\
\hline Pseudomyrmex sp. 1 & $\mathrm{X}$ & & $\mathrm{X}$ & & & & \\
\hline
\end{tabular}

Table 2. Observed (Sobs) and estimated (estimators: Jackknife 1 - Jack1 and Bootstrap - Boot) richness and number of exclusive species by method (Pitfall and TSBF) in Santa Catarina.

\begin{tabular}{lcccc}
\hline Methods & Sobs & Jack1 & Boot & Exclusive \\
\hline Pitfall & 202 & 260 & 227 & 89 \\
TSBF & 149 & 213 & 176 & 36 \\
Total & 238 & 294 & 323 & - \\
\hline
\end{tabular}

In Paraná, the TSBF method collected 101 species (Table 1), with 44, 88 and 52 species at strata 0, I and II (Table 3), and 97, 301 and 121 occurrence records, respectively. Four out of the 101 species were collected exclusively in stratum 0,30 only in stratum I and five in stratum II (Table 1). The most frequent species were Hypoponera sp. 1, Brachymyrmex sp. 5 and Pheidole cavifrons Emery, 1906, with 53, 25 and 16 occurrence records. Species occurrences in strata were as follows: in litter (stratum 0), the three most frequent species were Hypoponera sp. 1, with 12 records; Brachymyrmex sp. 1, and Brachymyrmex sp. 5, with seven records each. In stratum I (0-10 cm), the most frequent species were Hypoponera $\mathrm{sp}$. 1, Hypoponera $\mathrm{sp}$. 3 and Brachymyrmex sp. 3, with 29, 17 and 14 records, respectively. In stratum II $(10-20 \mathrm{~cm})$, the highest frequency was recorded for Hypoponera sp. 1, Brachymyrmex sp. 5 and Pheidole cavifrons, with 12,8 and 7 records.

Table 3. Observed richness (Sobs) and estimated (estimators: Jackknife 1 - Jack1 E Bootstrap - Boot) and number of exclusive species by strata (0 - litter, I - 0-10 $\mathrm{cm}$, and II- $10-20 \mathrm{~cm}$ ).

\begin{tabular}{lcccc}
\hline Strata & Sobs & Jack1 & Boot & Exclusive \\
\hline 0 & 44 & 66 & 54 & 4 \\
I & 88 & 125 & 106 & 30 \\
II & 53 & 77 & 64 & 5 \\
Total & 101 & 129 & 114 & - \\
\hline
\end{tabular}

The collector curves constructed for each method demonstrate that a greater effort would be required to reach the asymptote (although this is apparently close), and that the number of accumulated species per sample unit was greater in pitfall than in TSBF (Figure 4a). Epigaeic pitfall collected $77 \%$ and TSBF $70 \%$ of the numbers estimated by Jackknife 1 and $89 \%$ and $84 \%$ of the numbers estimated by Bootstrap (Table 2).

The collector curves for strata performed with data from Paraná show that under the same sampling effort there is a greater addition of species in stratum I, in relation to the others (Figure $4 b$ ). The numbers of species observed approximate the Bootstrap estimated values (Table 3). The species collected in each stratum correspond to $66 \%, 70 \%$ and $69 \%$ of the species estimated by Jackknife 1 (for strata 0 , I and II respectively) and $81 \%, 83 \%$ and $83 \%$ of the species estimated by Bootstrap. The total number of species corresponds to $78 \%$ and $88 \%$ of the estimate by Jackknife 1 and Bootstrap, respectively.

\section{Subterranean ant assembly characterization}

In the regions of Santa Catarina, we obtained 89 species exclusively in the epigaeic stratum and 36 species in the subterranean stratum. Seven species were recorded for the first time in the Plateau region of Santa Catarina and were collected only by TSBF: Acropyga decedens (Mayr, 1887), Fulakora armigera (Mayr, 1887), Gnamptogenys reichenspergeri (Santschi, 1929), Neocerapachys splendens (Borgmeier, 1957), Proceratium brasiliense Borgmeier, 1959, Sphinctomyrmex stali Mayr, 1866 and Typhlomyrmex major Santschi, 1923. No new records were found for the western region. Fifty-eight species were collected in Pitfall traps only once, while single species occurrences in TSBF samples totalled 64 . These numbers correspond to different proportions $\left(\chi^{2}=7.05, \mathrm{p}=0.007, \mathrm{gl}=1\right)$, with $28.7 \%$ and $42.9 \%$, respectively, of the total species collected by each method.

In Paraná, where the method utilised was exclusively TSBF, seven species were present only in stratum I: Fulakora elongata (Santschi, 1912), Gnamptogenys reichenspergeri, Oxyepoecus plaumanni Kempf 1974, Pheidole radozskowskii Mayr, 1884, Pseudomyrmex gracilis 


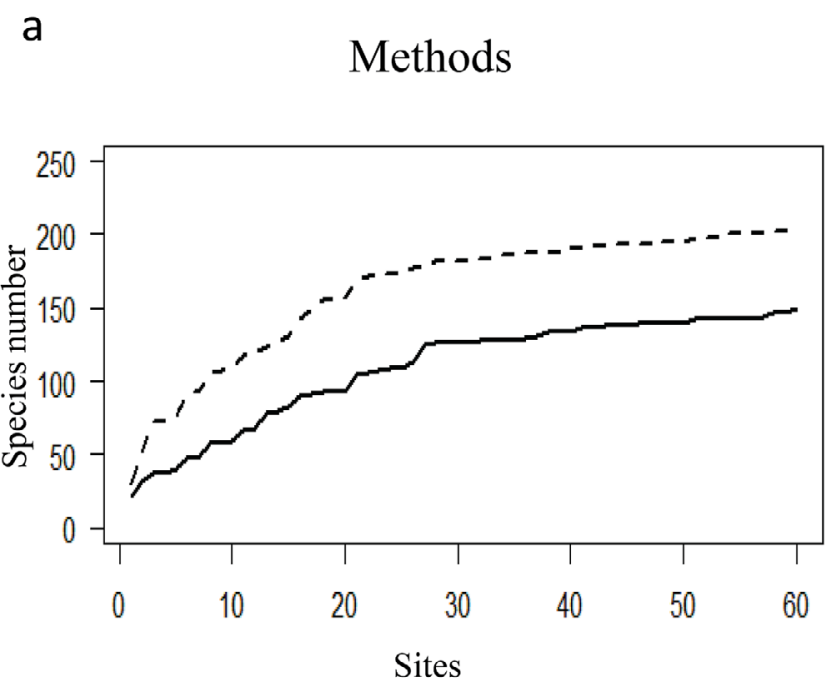

b

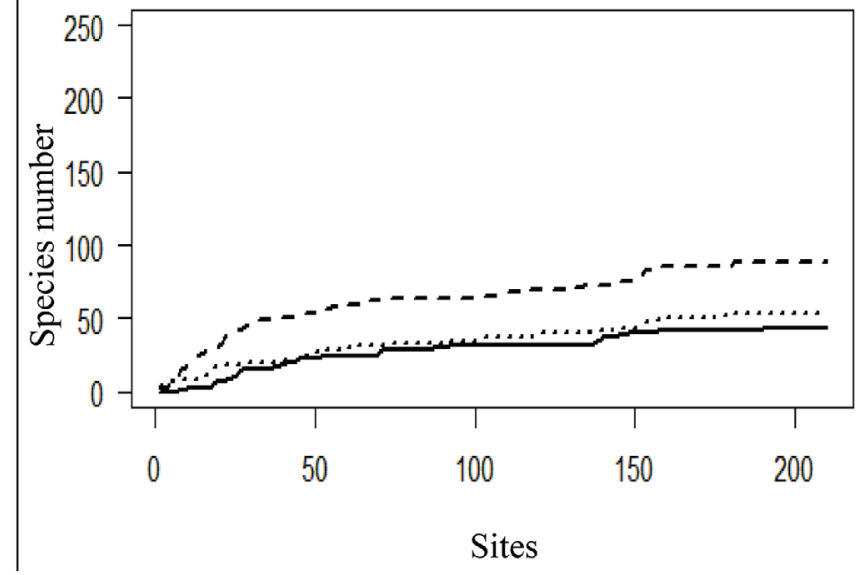

Figure 4. a - Collector's curves for both methods (Pitfall and TSBF). Dashed line: Pitfall; and continuous line: TSBF. b - Collector's curve for the strata. Continuous line: stratum 0; dashed line: stratum I; and dotted line: stratum II.

(Fabricius, 1804), P. longus (Forel, 1912) and Tranopelta gilva Mayr, 1866. In stratum II, Neivamyrmex sp. 1 was the only species (with only one occurrence).

The number of species with only one record of occurrence in strata was 22 for stratum 0, 37 for stratum I and 24 for stratum II (Figure 5a, b, and c). These species correspond to $50 \%, 42.1 \%$ and $46.1 \%$ of the total species collected in each stratum. The graph of observed richness (Sobs) and frequency of occurrence per stratum (Figure 5d) shows that in stratum I there is a great number of species that occur more frequently. Stratum I had the highest number of occurrence frequencies, once this is the stratum with the highest number of occurrences of ants (GLMM, Binomial, $\mathrm{z}=5,239, \mathrm{df}=4, \mathrm{p}<0.05)$, with a probability of occurrence of $86.10 \%$ in the sampled areas.

\section{Discussion}

The present study delimited and characterized subterranean ant assemblages in the subtropical regions of the Neotropics. In these regions, only two previous studies collected subterranean ants: (1) Silva \& Silvestre (2004) in Western Santa Catarina, and (2) Schmidt \& Diehl (2008) in Rio Grande do Sul. This is the first survey that evaluates subterranean ant assemblages in Paraná and the Plateau region of Santa Catarina. Despite the different sampling efforts, the aforementioned publications presented 32 and seven species belonging to the subterranean stratum, representing only $28 \%$ and $20 \%$ of the total species observed by those authors, respectively. Considering the number of species with restricted occurrence in the subterranean stratum in all sampled localities, our results were similar (in percentage): 57 species, or $20.2 \%$ of the total collected (Table 1 ).

The biology of most subterranean species is unknown. TSBF samples provide data of occurrence and preference among strata, nesting, and species composition in the different soil layers. The manual extraction of ants in the stratified monoliths, characteristic of this method, provides the opportunity for in loco observation of the collected organisms. Wong \& Guénard (2017) emphasized the importance of the "observer bias" (represented by different levels of experience in active collections), in which a collector may underestimate the presence of subterranean ants (especially the small ones) in samples or even in the field. This error can be reduced if sorting is performed under a stereomicroscope.

The total number of species observed in each municipality of Santa Catarina is extremely close to that observed in other studies already conducted in those regions (see Silva \& Silvestre 2004, Ulysséa et al. 2011, Lutinski \& Morais 2013). However, the number of species collected in Paraná is considerably high when compared with the richness found by other studies employing different methods for sampling subterranean ants (see Table 1 and Wong \& Guénard 2017). This could indicate a higher efficiency of TSBF in sampling subterranean ants in these areas. However, additional studies comparing methods for collecting hypogaeic ants are indispensable to confirm this tendency. These comparisons should especially consider the most practical and widely employed method currently, the hypogaeic pitfall traps (Schmidt \& Solar, 2010).

When epigaeic pitfall traps and TSBF are compared, it becomes clear that pitfall traps capture a greater number of species that have a high frequency of occurrence. However, when evaluating the number of infrequent species, TSBF is superior ( $42.7 \%$ of the species sampled). This may be explained by the fact that pitfall traps remained in the field for 72 hours, increasing the likelihood of species occurring more than once and increasing the number of species captured (see Lasmar et al 2017). Another factor that can explain this difference is the higher ant activity in the ground surface due to nesting and foraging than in the hypogaeic stratum (Jacquemin et al. 2016). In addition, in Santa Catarina, 36 species were collected exclusively by TSBF. This means that $15 \%$ of the species sampled there in this study would not have been registered without an association between the pitfall and a specific sampling method for the subterranean stratum. 
a

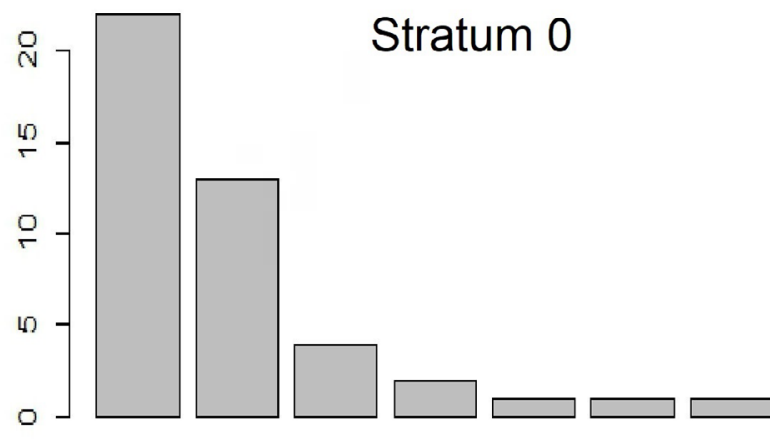

C

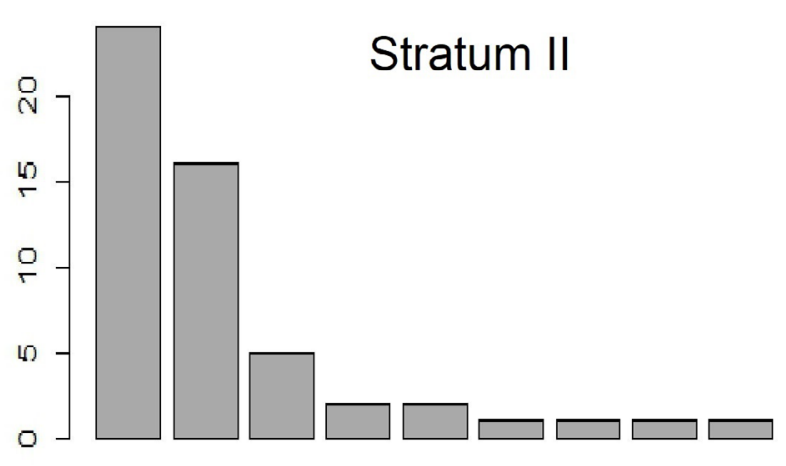

b

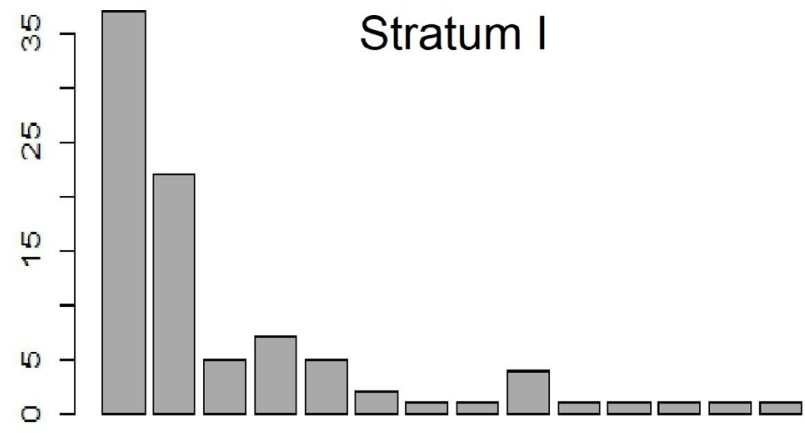

d

\section{Richness $x$ Frequency of occurrence}

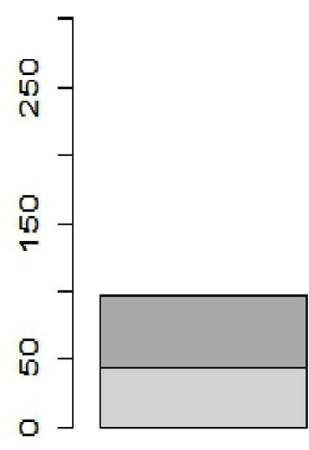

0

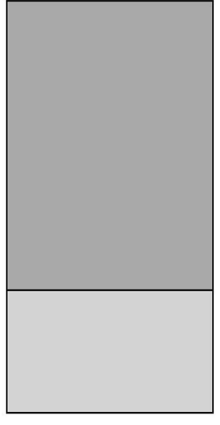

। $\square$ Frequency

$\square$ Richness

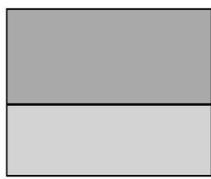

$\|$

\section{Strata}

Figure 5. a to c - Fisher graphs for each stratum sampled in Paraná; d - Comparative graph of observed richness (Sobs) and frequency of occurrence between the strata.

The results obtained by the GLMM analysis of the Paraná data show that stratum I has more species than the other strata. A similar result was found by Andersen \& Brault (2010) and Rider Wilkie et al. (2007), who also obtained a greater number of species in the topsoil and a great number of exclusive species (19 species) in this layer (up to $12,5 \mathrm{~cm}$ ). These results indicate a tendency for greater richness and frequency of occurrence in this stratum, which consequently must be considered during the sampling design of projects focusing on subterranean ants or even in comprehensive inventories of the ant fauna.

Sampling methods that record assemblages in different vertical strata allow for a more efficient evaluation of the association between ants and micro habitats. This association was effective for the delimitation of species that occur in different strata also in Jacquemin et al. (2016) and in the present work. The criterion of exclusive delimitation of occurrence used here proved to be efficient, as observed by Fowler et al. (2000). Most species identified by these authors belonged to a unique stratum (collected only in epigaeic traps or only in hypogaeic traps). Considering the exclusivity of occurrence in the strata, and collection methods (pitfall and TSBF), 33\% of the total species are epigaeic and $20 \%$ are hypogaeic.

More than a quarter (28.5\%) of the species identified in this study are subterranean and most can be considered infrequent. We thus consider the criterion "exclusivity" (stratum and/or method) a good approach for delimiting the subterranean habit, as already mentioned in literature (e.g. Fowler et al. 2000). However, the delimitation of subterranean ant species was possible only with the association of epigaeic and hypogaeic sampling methods. In addition, we found that in stratified TSBF samples the $0-10 \mathrm{~cm}$ stratum has the greatest number of species 
and highest frequency of occurrence. This fact, allied to the number of species obtained through sampling by the TSBF method, indicates that this is a useful method for sampling subterranean ants.

The localities sampled in this study are under intense pressure for urbanization and agricultural production. Thus, TSBF is a promising tool in diversity assessments (because it is a widely used method in soil quality studies, facilitating the acceptance by producers for the development of research in their areas), especially to access the impact of human activities on subterranean species. In addition, it was demonstrated that the TSBF captures rarely sampled species or species that are still undiscovered.

\section{Acknowledgments}

We thank Alexandre C. Ferreira, Gabriela P. Camacho, John E. Latke, Mayron Escárraga and Thiago S. R. da Silva for species identification. Special thanks to Sebastian F. Sendoya for the support in the data analyses. We thank IAPAR and Vila Velha State Park for technical support, and the Conselho Nacional de Desenvolvimento Cientifico e Tecnológico (CNPq) (Processes: 140260/2016; 563251/2010-7); Fundação de Amparo à Pesquisa e Inovação do Estado de Santa Catarina (FAPESC) (Process 6.309/2011-6/FAPESC) and Empresa Brasileira de Pesquisas Agropecuárias (Embrapa) (Project: 02.11.01.031.00.00) for the financial support. RMF was supported by CNPq (302462/2016-3). DB and GGB thank the CNPq for Research Productivity Grants (307162/2015-0, 304084/2010-7, 307486/2013-3). Collecting was done with permits from ICMBio (18128-1), IAP (463.12) and IBAMA (02027.002353/05-94).

\section{Author Contributions}

Mila Ferraz de Oliveira Martins: contributed substantially in the concept of the study, in data collection, data analysis and interpretation, and to prepare the manuscript.

Marcílio José Thomazini: contributed in the concept and design of the study; data collection, and to critical revision, adding intellectual content.

Dilmar Baretta: did a substantial contribution in the concept and design of the study, data collection, contributed to data analysis and interpretation and did a critical revision, adding intellectual content

Marcio Gonçalves da Rosa: contributed in the concept and design of the study, data collection, data analysis and interpretation, and did a critical revision, adding intellectual content

Maurício Rumenos Zagatto: contributed in the concept and design of the study, and data collection.

Alessandra Santos: contributed in the concept and design of the study, and data collection.

Herlon S. Nadolny: contributed in data collection

Guilherme B. X. Cardoso: contributed in data collection

Cintia C. Niva: contributed in data collection

Marie L. C. Bartz: contributed in the concept and design of the study, and data collection.

Rodrigo M. Feitosa: contributed substantially in the concept of the study, in data collection, data interpretation, and to prepare the manuscript.

\section{Conflicts of interest}

The authors declare that they have no conflict of interest related to the publication of this manuscript.

\section{References}

ANDERSEN, A. \& BRAULT, A. 2010. Exploring a new biodiversity frontier: subterranean ants in northern Australia. Biodivers Cons 19(9): 2741-2750.

ANDERSON, J.M. \&INGRAM, J.S.I. (ed). 1993. Tropical soil biology and fertility: a handbook of methods. 2nd ed. C.A.B. International, Wallingford.

BACCARO, F.F., FEITOSA, R.M., FERNÁNDEZ, F. et al. 2015. Guia para os Gêneros de formigas do Brasil. Editora INPA, Manaus.

BARTZ, M.L.C., BROWN, G.G., ROSA, M.G. et al. 2014a. Earthworm richness in land-use systems in Santa Catarina, Brazil. Appl Soil Ecol. 83: 59-70. DOI: http://dx.doi.org/10.1016/j.apsoil.2014.03.003

BARTZ, M.L.C., BROWN, G.G., ORSO, R., MAFRA, A.L., \& BARETTA, D. 2014b. The influence of land use systems on soil and surface litter fauna in the western region of Santa Catarina. Rev Ciên Agr. 45(5): 880-887. URL: http://ccarevista.ufc.br/seer/index.php/ccarevista/article/view/3453/1050

BENAZZI, E.D.S., BIANCHI, M.D.O., CORREIA, M.E.F., LIMA, E. \& ZONTA, E. 2013. Impactos dos métodos de colheita da cana-de-açúcar sobre a macrofauna do solo em área de produção no Espírito Santo - Brasil. Sem: Ciên Agr 34(6): 3425-3442. URL: http://www.uel.br/revistas/uel/index.php/ semagrarias/article/viewFile/12760/13670

BESTELMEYER, B., AGOSTI, D., ALONSO, L. et al. 2000. Field Techniques for the study of ground-dwelling ants: an overview, description and evaluation. pp: 122-144. In: D. AGOSTI, J. MAJER, L. ALONSO \& T. SCHUTZ, (eds). Ants- Standard methods for measuring and monitoring biodiversity, 1st ed. Smithsonian Institution Press, Washington and London.

BOTTINELLI, N., JOUQUET, P., CAPOWIEZ, Y. et al. 2015. Why is the influence of soil macrofauna on soil structure only considered by soil ecologists? Soil Till Res. 146: 118-124.

BRANDÃO, C.R.F., SILVA, R.R. \& FEITOSA, R.M. 2011. Cerrado grounddwelling ants (Hymenoptera: Formicidae) as indicators of edge effects. Zool. 28(3): 379-387. DOI: http://dx.doi.org/10.1590/S1984-46702011000300012

CORREIA, D.S. 2010. Fauna edáfica como indicadora em ambiente reconstruído após mineração de carvão. M. S. thesis, Universidade Estadual de Santa Catarina (UDESC). URL: http://tede.udesc.br/tede/tede/2228

DELABIE, J. \& FOWLER, H. 1995. Soil and litter cryptic ant assemblages of Bahia cocoa plantations. Pedobiol. 39(1): 423-433.

FOLGARAIT, P.J. 1998. Ant biodiversity and its relationship to ecosystem functioning: a review. Biodivers Cons. 7(9): 1221-1244. URL: https://link. springer.com/article/10.1023/A:1008891901953

FOWLER, H., DELABIE, J. \& MOUTINHO, P. 2000. Hypogaeic and epigaeic ant (Hymenoptera: Formicidae) assemblages of atlantic costal rainforest and dry mature and secondary Amazon forest in Brazil: continuums or communities. Trop Ecol. 41(1): 73-80.

GUTIÉRREZ, J.A.M., ROUSSEAU, G.X., ANDRADE-SILVA, J. \& DELABIE, J.H.C. 2017. Taxones superiores de hormigas como sustitutos de la riqueza de espécies en una cronosecuencia de bosques secundarios, bosque primario y sistemas agroforestales en la Amazonia Oriental, Brasil. Rev Biol Trop. 65(1): 279-291.

(IAPAR) Instituto Agronâmico do Paraná. 1978. Cartas climáticas básicas do estado do Paraná. Fundação Instituto Agronômico do Paraná. Londrina.

(IBGE) Instituto Brasileiro de Geografia e Estatística. 2016. Shapefiles for Brazil and Southern States [IBGE $>$ Bases e referenciais $>$ Malhas Digitais $>2015$ > Brasil/UFs]. URL: http://mapas.ibge.gov.br/bases-e-referenciais/ bases-cartograficas/malhas-digitais.html

JACQUEMIN, J., DROUET, T., DELSINNE, T., ROISIN, Y. \& LEPONCE, M. 2012. Soil properties only weakly affect subterranean ant distribution at small spatial scales. Appl Soil Ecol. 62:163-169.

JACQUEMIN, J., ROISIN, Y. \& LEPONCE, M. 2016. Spatio-temporal variation in ant (Hymenoptera: Formicidae) communities in leaf-litter and soil layers in a premontane subtropical forest. Myrmecol. News 22: 129-139. 
KLENK, L.A., ZAWADNEAK, M.A.C. \& BUCH, A.C. 2009. Cupins, Formigas e Minhocas como Indicadores de Recuperação da Qualidade de Solo sob Processo de Conversão - Pinhais (PR). Rev Bras Agroecol. 4(2): 3571-3575.

LASMAR, C., QUEIROZ, A., RABELLO, A. et al. 2017. Testing the effect of pitfall-trap installation on ant sampling. Insect Soc. 64(3): 445-451. DOI 10.1007/s00040-017-0558-7

LAVELLE, P., BIGNELL, D., LEPAGE, M. \& DHILLION, S.P. 1997. Soil function in a changing world: The role of invertebrate ecosystem engineers. Eur J Soil Biol. 33: 159-193.

LIMA, S.S., AQUINO, A.M., LEITE, L.F.C., VELÁSQUEZ, E. \& LAVELLE, P. 2010. Relação entre macrofauna edáfica e atributos químicos do solo em diferentes agroecossistemas. Pesqui Agropecu Bras. 45(3): 322-331. DOI: http://dx.doi.org/10.1590/S0100-204X2010000300013

LONGINO, J. 2000. What to do with the data. pp: 186-203. In: D. AGOSTI, J. MAJER, L. ALONSO \& T. SCHUTZ, (eds), Ants- Standard methods for measuring and monitoring biodiversity, 1st ed. Smithsonian Institution Press, Washington and London.

LOURENTE, E.R.P., SILVA, R.F., SILVA, D.A., MARCHETTI, M.E. \& MERCANTE, F.M. 2007. Macrofauna edáfica e sua interação com atributos químicos e físicos do solo sob diferentes sistemas de manejo. Acta Sci-Agr. 29(1), 17-22.

LUCKY, A., TRAUTWEIN, M., GUÉNARD, B., WEISSER, M. \& DUNN, R. 2013 Tracing the rise of ants - Out of ground. PLOS one 8(12): 1-8. URL: https://doi.org/10.1371/journal.pone.0084012

LUTINSKI, J.A., LOPES, B.C. \& MORAIS, A.B.B. 2013. Diversidade de formigas urbanas (Hymenoptera: Formicidae) de dez cidades do sul do Brasil. Biota Neotrop. 13(3): 332-342. DOI: 10.1590/S167606032013000300033

MAACK, R. 2012. Geografia Física do Estado do Paraná. 4th Ed. Universidade Estadual de Ponta Grossa, Ponta Grossa.

PEREIRA, R.C., ALBANEZ, J.M. \& MAMÉDIO, I.M.P. 2012. Diversidade da meso e macrofauna edáfica em diferentes sistemas de manejo de uso do solo em Cruz das Almas - BA. Magistra 24: 63-76.

QGIS Development Team. 2016. QGIS Geographic Information System. Open Source Geospatial Foundation. URL: https://www.qgis.org/en/site/
ROSA, M. G. 2013. Macrofauna do solo em diferentes sistemas de uso no oeste e planalto catarinense, M.S. thesis. Universidade Estadual de Santa Catarina (UDESC), Chapecó. URL: http://www.cav.udesc.br/arquivos/id submenu/832/marcio goncalves da rosa osmar klauber_filho_24 0.pdf

ROSA, M.G., KLAUBERG FILHO, O., BARTZ, M.L.C., MAFRA, A.L., SOUSA, J.P.F.A. \& BARETTA, D. 2015. Macrofauna Edáfica e Atributos Físicos e Químicos em Sistemas de Uso do solo no Planalto Catarinense. Rev Bras Cien Solo, 39(6): 1544-1553. URL: http://www.scielo.br/pdf/ rbcs/v39n6/0100-0683-rbcs-39-6-1544.pdf

ROUSSEAU, G.X., SILVA, P.R.S. \& CARVALHO, C.J.R. 2010. Earthworms, ants and other arthropods as soil health indicators in traditional and no-fire agro-ecosystems from eastern brazilian Amazonia. Acta Zool Mex. Número Especial 2: 117-134.

RYDER WILKIE, K., MERTL, A. \& TRANIELLO, J. 2007. Biodiversity below ground: probing the subterranean ant fauna of Amazonia. Naturwis. 94(9): 725-731.

SCHMIDT, F.A. \& DIEHL, E. 2008. What is the effect of soil use on ant communities? Neotrop Entomol. 37(4): 381-388. DOI: 10.1590/S1519566X2008000400005

SCHMIDT, F., RIBAS, C. \& SCHOEREDER, J. 2013. How predictable is the response of ant assemblages to natural forest recovery? Implications for their use as bioindicators. Ecol Indic. 24: 158-166.

SILVA, R.R. \& SILVESTRE, R. 2004. Riqueza da fauna de formigas (Hymenoptera: Formicidae) que habita as camadas superficiais do solo em Seara, Santa Catarina. Pap Avul Zool. 44(1): 1-11. URL: http://www. scielo.br/scielo.php?script=sci_arttext\&pid=S0031-10492004000100001

ULYSSÉA, M.A., CERETO, C.E., ROSUMEK, F.B., SILVA, R.R. \& LOPES, B.C. 2011. Updated list of ant species (Hymenoptera, Formicidae) recorded in Santa Catarina State, southern Brazil, with a discussion of research advances and priorities. Rev Bras Entomol. 55(4): 603-611. DOI: 10.1590/ S0085-56262011000400018

WONG, M. \& GUÉNARD, B. 2017. Subterranean ants: summary and perspectives on field sampling methods, with notes on diversity and ecology (Hymenoptera: Formicidae). Myrmecol News 25: 1-16.

ZAGATTO, M.R.G. 2014. Fauna edáfica em sistemas de uso do solo no município de Ponta Grossa- PR. M. S. thesis, Universidade Federal do Paraná (UFPR). URL: http://dspace.c3sl.ufpr.br/dspace/bitstream/handle/1884/35923/R\%20 -\%20D\%20-\%20MAURICIO\%20RUMENOS\%20GUIDETTI\%20 ZAGATTO.pdf?sequence $=1$ 